Вронська Людмила Вікторівна, кандидат хімічних наук, доцент, кафедра фармації, Навчальнонауковий інститут післядипломної освіти, ДВНЗ «Тернопільський державний медичний університет імені І. Я. Горбачевського МОЗ України», майдан Волі, 1, м. Тернопіль, Україна, 46001

E-mail: vronska_liudmyla@ukr.net

Грошовий Тарас Андрійович, доктор фармацевтичних наук, професор, завідувач кафедри, кафедра управління та економіки фармації з технологією ліків, ДВНЗ «Тернопільський державний медичний університет імені І. Я. Горбачевського МОЗ України», майдан Волі, 1, м. Тернопіль, Україна, 46001 E-mail: grochovuy.tern@mail.ru

UDC 547,792:547,856

DOI: $10.15587 / 2313-8416.2016 .65166$

\title{
THE SYNTHESIS, COMPUTER PREDICTION OF THE BIOLOGICAL ACTIVITY AND THE ACUTE TOXICITY OF 4-ARYL-5-OXO-4,5-DIHYDRO[1,2,4]TRIAZOLO[4,3- $a]$ QUINAZOLINE-8-CARBOXAMIDES
}

\author{
(C) S. Danylchenko, O. Drushlyak, S. Kovalenko, S. Kovalenko
}

Aim. The aim of present study was to conduct modelling of the virtual library of 4-aryl-5-oxo-4,5-dihydro[1,2,4]triazolo[4,3-a]quinazoline-8-carboxamides, to determine the most probable biological activity spectrum and the acute toxicity of studied compounds by PASS and GUSAR software, sort out the most perspective substances and develop preparative protocols for their synthesis.

Methods. Using the PASS program computer prediction of the biological activity of 4-aryl-5-oxo-4,5dihydro[1,2,4]triazolo[4,3-a]quinazoline-8-carboxamides has been performed. Prediction of the acute toxicity has been carried out by the GUSAR software. The structure of the compounds synthesized has been proven by elemental analysis and ${ }^{1} H$ NMR spectroscopy data.

Results. The synthesis of 4-aryl-5-oxo-4,5-dihydro[1,2,4]triazolo[4,3-a]quinazoline-8-carboxamides has been conducted starting from corresponding methyl 3-aryl-4-oxo-2-thioxo-1,2,3,4-tetrahydroquinazoline-7-carboxylates, which were converted into corresponding 3-aryl-2-hydrazino-4-oxo-3,4-dihydroquinazoline-7-carbohydrazides by treatment with hydrazine hydrate. Heating of these 2-hydrazinoquinazolin-4(3H)-ones with acetylacetone was resulted in 4-aryl-8-[(3,5-dimethyl-1H-pyrazol-1-yl)carbonyl]-1-methyl[1,2,4]triazolo[4,3-a]quinazolin-5(4H)-ones formation. Following substitution of pyrazole moiety by interaction of these compounds with primary amines led to destinated 4-aryl-5-oxo-4,5-dihydro[1,2,4]triazolo[4,3-a]quinazoline-8-carboxamides. The PASS program computer prediction of the biological activity of 4-aryl-5-oxo-4,5-dihydro[1,2,4]triazolo[4,3a]quinazoline-8-carboxamides has allowed identifying the types of activity of studied compounds and sorting out the leaders with potential antineurotic activity, which are perspective for male reproductive and erectile dysfunction treatment. Prediction of the acute toxicity has been carried out by the GUSAR software, which allowed to refer them to slightly toxic (class 4 ) or practically nontoxic (class 5) substances.

Conclusions. The obtained compounds are perspective objects for further investigations as slightly toxic (nontoxic) substances with potential antineurotic activity, which are perspective for male reproductive and erectile dysfunction treatment.

Keywords: synthesis, 4-aryl-5-oxo-4,5-dihydro[1,2,4]triazolo[4,3-a]quinazoline-8-carboxamides, computer prediction, biological activity, acute toxicity

Мета. У даному дослідженні було поставлено за мету провести моделювання віртуальної бібліотеки 4-арил-5-оксо-4,5-дигідро[1,2,4]триазоло[4,3-а]хіназолін-8-карбоксамідів, за допомогою комп'ютерних програм PASS та GUSAR визначити найбільш ймовірний спектр біологічної активності та гостру токсичність досліджуваних сполук, а також виділити найбільш перспективні сполуки та розробити препаративні методи їх синтезу.

Методи. Комп'ютерне прогнозування біологічної активності 4-арил-5-оксо-4,5-дигідро[1,2,4]триазоло[4,3-а]хіназолін-8-карбоксамідів проведено за допомогою програми PASS. Комп'ютерне прогнозування гострої токсичності здійснено за програмним забезпеченням GUSAR. Будову синтезованих сполук доведено за допомогою елементного аналізу та даних ${ }^{1}$ H ЯMP спектроскопії. 
Результати. Синтез 4-арил-5-оксо-4,5-дигідро[1,2,4]триазоло[4,3-а]хіназолін-8-карбоксамідів був проведений виходячи з відповідних метил 3-арил-4-оксо-2-тіоксо-1,2,3,4-тетрагідрохіназолін-7-карбоксилатів, які були перетворені у відповідні 3-арил-2-гідразино-4-оксо-3,4-дигідрохіназолін-7-карбогідразиди під дією гідразин гідрату. Нагрівання ичи 2-гідразинохіназолін-4(3Н)-онів в ацетилацетоні привело до утворення 4-арил-8-[(3,5-диметил-1Н-піразол-1-іл)карбоніл]-1-метил[1,2,4]триазоло[4,3а]хіназолін-5(4H)-онів. Наступне заміщення залишку піразолу при взаємодї цих сполук з первинними амінами дало цільові 4-арил-5-оксо-4,5-дигідро[1,2,4]триазоло[4,3-а]хіназолін-8-карбоксаміди. Комп'ютерне прогнозування біологічної активності 4-арил-5-оксо-4,5-дигідро[1,2,4]триазоло[4,3-а]хіназолін-8карбоксамідів за допомогою програми PASS дозволило визначити напрямок активності досліджуваних сполук та виділити серед них лідерів з потенційною антиневротичною активністю, які можуть бути перспективними для лікування чоловічих репродуктивних захворювань та еректильних дисфункиій. Комп'ютерне прогнозування гострої токсичності здійснено за програмним забезпеченням GUSAR, щчо дозволило віднести їх до малотоксичних (4 клас) або практично нетоксичних речовин (5 клас).

Висновки. Отримані сполуки є перспективними об'єктами для подальших досліджень як малотоксичні (нетоксичні) речовини з потенційною антиневротичною активністю, які можуть бути перспективними для лікування чоловічих репродуктивних захворювань та еректильних дисфункцій

Ключові слова: синтез, 4-арил-5-оксо-4,5-дигідро[1,2,4]триазоло[4,3-а]хіназолін-8-карбоксаміди, комn'ютерне прогнозування, біологічна активність, гостра токсичність

\section{Introduction}

Derivatives of $[1,2,4]$ triazolo[4,3- $a]$ quinazolin$5(4 H)$-one, which are representatives of the important class of condensed heterocycles possessing wide range of the biological activity, attract particular interest in development of innovative drug substances.

2. Formulation of the problem in a general way, the relevance of the theme and its connection with important scientific and practical issues

The possibility to synthesize a large amount of $[1,2,4]$ triazolo[4,3-a]quinazolin-5(4H)-one derivatives leads to the necessity for the rational presynthetic selection the most perspective compounds from defined variety. One of the effective ways to solve this problem is computer prediction of various properties of $[1,2,4]$ triazolo[4,3a]quinazolin-5(4H)-one derivatives, such as biological activity and acute toxicity, that enables to eliminate unpromising substances at the early stages of the research.

\section{Analysis of recent studies and publications in} which a solution of the problem and which draws on the author

Among potential pharmacologically significant properties of $[1,2,4]$ triazolo[4,3-a]quinazolin-5(4H)-one derivatives the H1-antihistaminic [1-9], anticonvulsant [10], antiHIV [11], antibacterial [11-13], antifungal [11, 12], antitubercular [11, 13], anticancer [13], anti-asthmatic [8, 14], antiallergic [14], anti-inflammatory [14, 15] bioactivities should be mentioned. In the previous study [16] we predicted potential antiasthmatic and antiallergic activity of compounds of the specified class.

4. Allocation of unsolved parts of the general problem, which is dedicated to the article

The presence of amide group may have a significant impact on biological behavior of compounds. Investigation of influence of amide moiety on the biological activity of $[1,2,4]$ triazolo[4,3-a]quinazolin-5(4H)-ones is important for expancion of knowledge about pharmacological properties of this class of compounds.

\section{Formulation of goals (tasks) of article}

Taking into account actuality of searching biological active substances among [1,2,4]triazolo[4,3-a]quinazolin-5(4H)-one derivatives and modern advances in software for virtual screening the goal of present study was to conduct modelling of the virtual library of 4-aryl5-oxo-4,5-dihydro[1,2,4]triazolo[4,3-a]quinazoline-8carboxamides, to determine the most probable biological activity spectrum and the acute toxicity of studied compounds by PASS and GUSAR software, sort out the most perspective substances and develop preparative protocols for their synthesis.

6. Statement of the basic material of the study (methods and objects) with the justification of the results

For design of the virual library of 4-aryl-5-oxo-4,5dihydro[1,2,4]triazolo[4,3-a]quinazoline-8-carboxamides 2 randomization points (aryl substituent in position 4 and different amine residue) and fixed methyl substituent in position 1 of $[1,2,4]$ triazolo[4,3-a]quinazolin-5(4H)-one were chosen.

The synthesis of studied 4-aryl-5-oxo-4,5-dihydro[1,2,4]triazolo[4,3-a]quinazoline-8-carboxamides was carried out by scheme, which were based on previously founded the formation of [1,2,4]triazolo[4,3-a]quinazolin-5(4H)-ones by reaction of 2-hydrazinoquinazolin$4(3 H)$-ones with acetylacetone (Fig. 1) [17]. Starting methyl 3-aryl-4-oxo-2-thioxo-1,2,3,4-tetrahydroquinazoline-7-carboxylates $\mathbf{1 a}, \mathbf{b}$ were converted into corresponding 2-hydrazinoquinazolin-4(3H)-ones $\mathbf{2 a , b}$ by treatment with hydrazine hydrate according improved method [18]. Heating of hydrazines 2a, b with acetylacetone was resulted in 4-aryl-8-[(3,5-dimethyl-1Hpyrazol-1-yl)carbonyl]-1-methyl[1,2,4]triazolo[4,3-a]quinazolin-5(4H)-ones 3a, b formation. Following substitution of pyrazole moiety by interaction of compounds 3a, b with primary amines led to destinated amides $4 \mathbf{a}-\mathbf{l}$. This way allows obtaining the library of final products in good yields. 
<smiles>[R1]n1c(=S)[nH]c2cc(C(=O)OC)ccc2c1=O</smiles>

1a, b<smiles>[R10]n1c(NN)nc2cc(C(=O)NN)ccc2c1=O</smiles>

2a, b

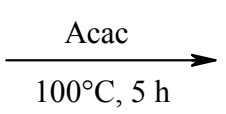<smiles>[R11]n1c(=O)c2ccc(C(=O)n3nc(C)cc3C)cc2n2c(C)nnc12</smiles>
reflux, $8 \mathrm{~h} \downarrow \mathrm{R}_{2} \mathrm{NH}_{2}, \mathrm{DMF}$<smiles>[R2]NC(=O)c1ccc2c(=O)n([R1])c3nnc(C)n3c2c1</smiles>

$\mathrm{R} 1=\mathrm{Ph}, 4-\mathrm{FPh}$

$\mathrm{R} 2=i-\mathrm{C}_{5} \mathrm{H}_{11}, 4-\mathrm{MeBn}, 4-\mathrm{MeOBn}, \mathrm{Ph}\left(\mathrm{CH}_{2}\right)_{3}, 3-\mathrm{MePh}, 3-\mathrm{MeOPh}$

Fig. 1. The synthesis of 4-aryl-5-oxo-4,5-dihydro[1,2,4]triazolo[4,3-a]quinazoline-8-carboxamides

The general procedure for the synthesis of 3 aryl-2-hydrazino-4-oxo-3,4-dihydroquinazoline-7-carbohydrazides (2a, b). Add the corresponding methyl 3-aryl-4-oxo-2-thioxo-1,2,3,4-tetrahydroquinazoline-7-carboxylate 1a, b $(0.05 \mathrm{~mol})$ to stirred mixture of dioxane $(100 \mathrm{ml})$ and hydrazine hydrate $(40 \mathrm{ml})$. Reflux the obtained emulsion with stirring for $3 \mathrm{~h}$. Separate the lower dioxane layer and dilute it with $\mathrm{H}_{2} \mathrm{O}(200 \mathrm{ml})$. Filter the precipitate, wash it with $i$-propanol $(100 \mathrm{ml})$ and recrystallize from mixture of DMF $(100 \mathrm{ml})$ and $i$-propanol $(200 \mathrm{ml})$. Yields, melting points, elemental analysis and ${ }^{1} \mathrm{H}$ NMR data are given in Table 1.

The general procedure for the synthesis of 4aryl-8-[(3,5-dimethyl-1H-pyrazol-1-yl)carbonyl]-1-methyl[1,2,4]triazolo[4,3-a]quinazolin-5(4H)-ones (3a, b). Heat the suspension of corresponding 3-aryl-2-hydrazino-4-oxo-3,4-dihydroquinazoline-7-carbohydrazi-de 2a, b $(0.03 \mathrm{~mol})$ in acetylacetone $(100 \mathrm{ml})$ at $100^{\circ} \mathrm{C}$ with stirring for 5 hours. After cooling dilute the reaction mixture with $i$ propanol $(300 \mathrm{ml})$. Filter the precipitate, wash it with $i$ - propanol $(50 \mathrm{ml})$ and recrystallize from mixture of DMF $(50 \mathrm{ml})$ and $i$-propanol $(100 \mathrm{ml})$. Yields, melting points, elemental analysis and ${ }^{1} \mathrm{H}$ NMR data are given in Table 1.

The general procedure for the synthesis of 4aryl-5-0xo-4,5-dihydro[1,2,4]triazolo[4,3-a]quinazoline8-carboxamides (4a-1). Reflux the solution of corresponding 4-aryl-8-[(3,5-dimethyl-1H-pyrazol-1-yl)carbonyl]-1-methyl[1,2,4]triazolo[4,3-a]quinazolin-5(4H)-one 3a, b $(0.002 \mathrm{~mol})$ in anhydrous DMF $(10 \mathrm{ml})$ and corresponding amine $(0.003 \mathrm{~mol})$ during 8 hours. After cooling dilute the reaction mixture with $\mathrm{H}_{2} \mathrm{O}(30 \mathrm{ml})$. Filter the precipitate formed, wash it with $i$-propanol $(10 \mathrm{ml})$ and recrystallize from mixture of DMF $(5 \mathrm{ml})$ and $i-$ propanol $(10 \mathrm{ml})$. Yields, melting points, elemental analysis and ${ }^{1} \mathrm{H}$ NMR data are given in Table 1.

The structures of obtained compounds 4a-l have been confirmed by the ${ }^{1} \mathrm{H}$ NMR spectroscopy data (Table 1). ${ }^{1} \mathrm{H}$ NMR-spectra were recorded on Varian WXR-400 (200 $\mathrm{MHz}$ ) spectrometer in DMSO-d6 solution with TMS as internal standard, chemical shifts are reported in ppm.

Properties of the compounds $\mathbf{2 a}, \mathbf{b}$; $\mathbf{3 a}, \mathbf{b} ; \mathbf{4 a - 1}$

\begin{tabular}{|c|c|c|c|c|c|c|c|c|}
\hline $\begin{array}{l}\text { Comp. } \\
\text { code }\end{array}$ & $\mathrm{R}$ & $\begin{array}{l}\text { Yield, } \\
\%\end{array}$ & $\underset{{ }^{\circ} \mathrm{C}}{\text { M.p., }}$ & $\begin{array}{l}\text { Molecular } \\
\text { formula, } \\
\text { m.m }\end{array}$ & $\begin{array}{l}\mathrm{C}, \% \\
\text { Calc./ } \\
\text { found }\end{array}$ & $\begin{array}{l}\mathrm{H}, \% \\
\text { Calc./ } \\
\text { found }\end{array}$ & $\begin{array}{l}\mathrm{N}, \% \\
\text { Calc./ } \\
\text { found }\end{array}$ & ${ }^{1} \mathrm{H}$ NMR spectral data $\delta$, ppm, J, hz \\
\hline 1 & 2 & 3 & 4 & 5 & 6 & 7 & 8 & 9 \\
\hline $2 \mathbf{a}$ & $\mathrm{R} 1=\mathrm{Ph}$ & 78 & $>300$ & $\begin{array}{c}\mathrm{C}_{15} \mathrm{H}_{14} \mathrm{~N}_{6} \mathrm{O}_{2} \\
310.31\end{array}$ & $\begin{array}{l}58.06 / \\
57.97\end{array}$ & $\begin{array}{l}4.55 / \\
4.58\end{array}$ & $\begin{array}{l}27.08 / \\
27.13\end{array}$ & $\begin{array}{c}4.55 \text { br.s, }\left(2 \mathrm{H}, \mathrm{CONHNH_{2 } )} ; 5.70 \mathrm{~s},(2 \mathrm{H},\right. \\
\left.\mathrm{NHNH}_{2}-2\right) ; 7.08 \mathrm{t},(\mathrm{J} 7.8,1 \mathrm{H}, \mathrm{H}-4 \mathrm{Ph}) ; \\
7.33 \mathrm{t},(\mathrm{J} 7.8,2 \mathrm{H}, \mathrm{H}-3,5 \mathrm{Ph}) ; 7.57 \mathrm{dd},\left(\mathrm{J}_{5,6}\right. \\
\left.7.8, \mathrm{~J}_{6,8} 2.0,1 \mathrm{H}, \mathrm{H}-6\right) ; 7.75 \mathrm{~d},\left(\mathrm{~J}_{6,8} 2.0,1 \mathrm{H},\right. \\
\mathrm{H}-8) ; 7.88 \mathrm{~d},(\mathrm{~J} 7.8,2 \mathrm{H}, 2,6 \mathrm{Ph}) ; 8.00 \mathrm{~d}, \\
\left(\mathrm{~J}_{5,6} 7.8,1 \mathrm{H}, \mathrm{H}-5\right) ; 9.40 \mathrm{~s},\left(1 \mathrm{H}, \mathrm{NHNH}_{2}-2\right) ; \\
\quad 10.02 \mathrm{~s},\left(1 \mathrm{H}, \mathrm{CON}_{\underline{\mathrm{HNH}}}\right)\end{array}$ \\
\hline $2 \mathbf{b}$ & $\begin{aligned} & \mathrm{R} 1= \\
= & 4-\mathrm{FPh}\end{aligned}$ & 80 & $>300$ & $\begin{array}{c}\mathrm{C}_{15} \mathrm{H}_{13} \mathrm{FN}_{6} \mathrm{O}_{2} \\
328.30\end{array}$ & $\begin{array}{l}54.88 / \\
54.92\end{array}$ & $\begin{array}{l}3.99 / \\
4.01\end{array}$ & $\begin{array}{l}25.60 / \\
25,57\end{array}$ & 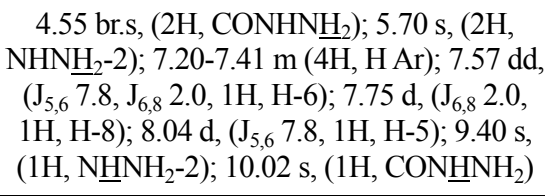 \\
\hline Зa & $\mathrm{R} 1=\mathrm{Ph}$ & 74 & $\begin{array}{l}296- \\
298\end{array}$ & $\begin{array}{c}\mathrm{C}_{22} \mathrm{H}_{18} \mathrm{~N}_{6} \mathrm{O}_{2} \\
398.42\end{array}$ & $\begin{array}{l}66.32 / \\
66.39\end{array}$ & $\begin{array}{l}4.55 / \\
4.52\end{array}$ & $\begin{array}{l}21.09 / \\
21.16\end{array}$ & $\begin{array}{c}2.12 \mathrm{~s},\left(3 \mathrm{H}, \mathrm{CH}_{3} \mathrm{Pyr}-3\right) ; 2.36 \mathrm{~s},(3 \mathrm{H}, \\
\left.\mathrm{CH}_{3}-1\right) ; 2.55 \mathrm{~s},\left(3 \mathrm{H}, \mathrm{CH}_{3} \mathrm{Pyr}-5\right) ; 6.26 \mathrm{~s}, \\
(1 \mathrm{H}, \mathrm{H} \mathrm{Pyr}-4) ; 7.52-7.76 \mathrm{~m},(6 \mathrm{H}, \mathrm{H}-7, \\
5 \mathrm{H} \mathrm{Ph}) ; 8.00 \mathrm{~d},\left(\mathrm{~J}_{7,9} 2.0,1 \mathrm{H}, \mathrm{H}-9\right) ; 8.31 \mathrm{~d}, \\
\left(1 \mathrm{H}, \mathrm{J}_{6,7} 7.8,1 \mathrm{H}, \mathrm{H}-6\right)\end{array}$ \\
\hline
\end{tabular}




\begin{tabular}{|c|c|c|c|c|c|c|c|c|}
\hline 1 & 2 & 3 & 4 & 5 & 6 & 7 & 8 & 9 \\
\hline 3b & $\begin{aligned} & \mathrm{R} 1= \\
= & 4-\mathrm{FPh}\end{aligned}$ & 78 & $\begin{array}{l}303- \\
304\end{array}$ & $\begin{array}{c}\mathrm{C}_{22} \mathrm{H}_{17} \mathrm{FN}_{6} \mathrm{O}_{2} \\
416.41\end{array}$ & $\begin{array}{l}63.46 / \\
63.51\end{array}$ & $\begin{array}{l}4.11 / \\
4.09\end{array}$ & $\begin{array}{l}20.18 / \\
20.22\end{array}$ & $\begin{array}{c}2.12 \mathrm{~s},\left(3 \mathrm{H}, \mathrm{CH}_{3} \mathrm{Pyr}-3\right) ; 2.36 \mathrm{~s},(3 \mathrm{H}, \\
\left.\mathrm{CH}_{3}-1\right) ; 2.55 \mathrm{~s},\left(3 \mathrm{H}, \mathrm{CH}_{3} \mathrm{Pyr}-5\right) ; 6.26 \mathrm{~s}, \\
(1 \mathrm{H}, \mathrm{H} \mathrm{Pyr}-4) ; 7.50 \mathrm{t}(\mathrm{J} 7.6,2 \mathrm{H}, \\
\mathrm{H}-3,5-\mathrm{Ar}) ; 7.70-7.82 \mathrm{~m},(3 \mathrm{H}, \mathrm{H} 7, \\
\mathrm{H}-2,6-\mathrm{Ar}) ; 8.04 \mathrm{~d},\left(\mathrm{~J}_{7,9} 2.0,1 \mathrm{H}, \mathrm{H}-9\right) ; \\
8.30 \mathrm{~d},\left(\mathrm{~J}_{6,7} 7.8,1 \mathrm{H}, \mathrm{H}-6\right)\end{array}$ \\
\hline $4 a$ & $\begin{array}{c}\mathrm{R} 1=\mathrm{Ph} \\
\mathrm{R} 2=i-\mathrm{C}_{5} \mathrm{H}_{11}\end{array}$ & 84 & $>300$ & $\begin{array}{c}\mathrm{C}_{22} \mathrm{H}_{23} \mathrm{~N}_{5} \mathrm{O}_{2} \\
389.45\end{array}$ & $\begin{array}{l}67.85 / \\
67.78\end{array}$ & $\begin{array}{l}5.95 / \\
5.99\end{array}$ & $\begin{array}{l}17.98 / \\
18.03\end{array}$ & $\begin{array}{c}0.88 \mathrm{~d},\left(\mathrm{~J} 7.0,6 \mathrm{H}, 2 \mathrm{CH}_{3}\right) ; 1.40 \mathrm{qr},(\mathrm{J} 7.0, \\
\left.2 \mathrm{H}, \mathrm{NHCH}_{2} \mathrm{CH}_{2}\right) ; 1.52-1.72 \mathrm{~m},(1 \mathrm{H}, \\
\left.\mathrm{C} \underline{\mathrm{H}}\left(\mathrm{CH}_{3}\right)_{2}\right) ; 2.36 \mathrm{~s},\left(3 \mathrm{H}, \mathrm{CH}_{3}-1\right) ; 3.25 \mathrm{qr}, \\
\left(\mathrm{J} 7.0,2 \mathrm{H}, \mathrm{NHCH}_{2} \mathrm{CH}_{2}\right) ; 7.42-7.56 \mathrm{~m}, \\
(5 \mathrm{H}, \mathrm{H} \mathrm{Ph}) ; 7.74 \mathrm{dd},\left(\mathrm{J}_{6,7} 7.8, \mathrm{~J}_{7,9} 2.0,\right. \\
1 \mathrm{H}, \mathrm{H}-7) ; 8.00 \mathrm{~d},\left(\mathrm{~J}_{7,9} 2.0,1 \mathrm{H}, \mathrm{H}-9\right) ; \\
8.26 \mathrm{~d},\left(\mathrm{~J}_{6,7} 7.8,1 \mathrm{H}, \mathrm{H}-6\right) ; 8.55 \mathrm{t}, \\
(\mathrm{J} 7.0,1 \mathrm{H}, \mathrm{CONH})\end{array}$ \\
\hline $4 b$ & $\begin{array}{c}\mathrm{R} 1=\mathrm{Ph} \\
\mathrm{R} 2= \\
=4-\mathrm{MeBn}\end{array}$ & 86 & $>300$ & $\begin{array}{c}\mathrm{C}_{25} \mathrm{H}_{21} \mathrm{~N}_{5} \mathrm{O}_{2} \\
423.47_{2}\end{array}$ & $\begin{array}{l}70.91 / \\
70.86\end{array}$ & $\begin{array}{l}5.00 / \\
4.97\end{array}$ & $\begin{array}{l}16.54 / \\
16.58\end{array}$ & $\begin{array}{c}2.24 \mathrm{~s}\left(3 \mathrm{H}, \mathrm{CH}_{3}\right) ; 2.36 \mathrm{~s},\left(3 \mathrm{H}, \mathrm{CH}_{3}-1\right) ; 4.40 \mathrm{~d} \\
\left(\mathrm{~J} 7.0,2 \mathrm{H}, \mathrm{CH}_{2}\right) ; 7.09 \mathrm{~d},(\mathrm{~J} 7.8,2 \mathrm{H}, 3,5 \mathrm{Bn}) ; \\
7.19 \mathrm{~d},(\mathrm{~J} 7.8,2 \mathrm{H}, 2,6 \mathrm{Bn}) ; 7.58-7.72 \mathrm{~m},(5 \mathrm{H}, \\
\mathrm{H} \mathrm{Ph}) ; 7.77 \mathrm{dd},\left(\mathrm{J}_{6,7} 7.8, \mathrm{~J}_{7,9} 2.0,1 \mathrm{H}, \mathrm{H}-7\right) ; \\
8.04 \mathrm{~d},\left(\mathrm{~J}_{7,9} 2.0,1 \mathrm{H}, \mathrm{H}-9\right) ; 8.26 \mathrm{~d},\left(\mathrm{~J}_{6,7} 7.8,\right. \\
1 \mathrm{H}, \mathrm{H}-6) ; 9.18 \mathrm{t},(\mathrm{J} 7.0,1 \mathrm{H}, \mathrm{CONH})\end{array}$ \\
\hline $4 c$ & $\begin{array}{c}\mathrm{R} 1=\mathrm{Ph} \\
\mathrm{R} 2= \\
=4-\mathrm{MeOBn}\end{array}$ & 85 & $>300$ & $\begin{array}{c}\mathrm{C}_{25} \mathrm{H}_{21} \mathrm{~N}_{5} \mathrm{O}_{3} \\
439.47\end{array}$ & $\begin{array}{l}68.33 / \\
68.39\end{array}$ & $\begin{array}{l}4.82 / \\
4.78 /\end{array}$ & $\begin{array}{l}15.94 / \\
15.89\end{array}$ & $\begin{array}{c}2.36 \mathrm{~s},\left(3 \mathrm{H}, \mathrm{CH}_{3}-1\right) ; 3.70 \mathrm{~s}\left(3 \mathrm{H}, \mathrm{OCH}_{3}\right) ; 4.37 \\
\mathrm{~d}\left(\mathrm{~J} 7.0,2 \mathrm{H}, \mathrm{CH}_{2}\right) ; 6.85 \mathrm{~d},(\mathrm{~J} 7.8,2 \mathrm{H}, 3,5 \\
\mathrm{Bn}) ; 7.22 \mathrm{~d},(\mathrm{~J} 7.8,2 \mathrm{H}, 2,6 \mathrm{Bn}) ; 7.58-7.72 \mathrm{~m}, \\
(5 \mathrm{H}, \mathrm{H} \mathrm{Ph}) ; 7.77 \mathrm{dd},\left(\mathrm{J}_{6,7} 7.8, \mathrm{~J}_{7,9} 2.0,1 \mathrm{H},\right. \\
\mathrm{H}-7) ; 8.04 \mathrm{~d},\left(\mathrm{~J}_{7,9} 2.0,1 \mathrm{H}, \mathrm{H}-9\right) ; 8.26 \mathrm{~d}, \\
\left(\mathrm{~J}_{6,7} 7.8,1 \mathrm{H}, \mathrm{H}-6\right) ; 9.18 \mathrm{t},(\mathrm{J} 7.0,1 \mathrm{H}, \mathrm{CONH})\end{array}$ \\
\hline $4 d$ & $\begin{array}{c}\mathrm{R} 1=\mathrm{Ph} \\
\mathrm{R} 2= \\
=\mathrm{Ph}-\left(\mathrm{CH}_{2}\right)_{3}\end{array}$ & 75 & $\begin{array}{l}282- \\
284\end{array}$ & $\begin{array}{c}\mathrm{C}_{26} \mathrm{H}_{23} \mathrm{~N}_{5} \mathrm{O}_{2} \\
437.49\end{array}$ & $\begin{array}{l}71.36 / \\
71.40\end{array}$ & $\begin{array}{l}5.30 / \\
5.28\end{array}$ & $\begin{array}{l}16.01 / \\
15.98\end{array}$ & $\begin{array}{r}1.80 \mathrm{qn},\left(\mathrm{J} 7.0,2 \mathrm{H}, 2-\mathrm{CH}_{2}\right) ; 2.36 \mathrm{~s},(3 \mathrm{H}, \\
\left.\mathrm{CH}_{3}-1\right) ; 2.59 \mathrm{t},\left(\mathrm{J} 7.0,2 \mathrm{H}, 3-\mathrm{CH}_{2}\right) ; 3.25 \mathrm{qr}, \\
\left(\mathrm{J} 7.0,2 \mathrm{H}, 1-\mathrm{CH}_{2}\right) ; 7.15-7.30 \mathrm{~m},(5 \mathrm{H}, \\
\mathrm{H} \mathrm{Ph}-3) ; 7.42-7.56 \mathrm{~m},(5 \mathrm{H}, \mathrm{H} \mathrm{Ph}) ; 7.74 \mathrm{dd}, \\
\left(\mathrm{J}_{6,7} 7.8, \mathrm{~J}_{7,9} 2.0,1 \mathrm{H}, \mathrm{H}-7\right) ; 8.00 \mathrm{~d},\left(\mathrm{~J}_{7,9} 2.0,\right. \\
1 \mathrm{H}, \mathrm{H}-9) ; 8.26 \mathrm{~d},\left(\mathrm{~J}_{6,7} 7.8,1 \mathrm{H}, \mathrm{H}-6\right) ; 8.66 \mathrm{t}, \\
(\mathrm{J} 7.0,1 \mathrm{H}, \mathrm{CONH})\end{array}$ \\
\hline $4 e$ & $\begin{array}{c}\mathrm{R} 1=\mathrm{Ph} \\
\mathrm{R} 2=3-\mathrm{MePh}\end{array}$ & 74 & $>300$ & $\begin{array}{c}\mathrm{C}_{24} \mathrm{H}_{19} \mathrm{~N}_{5} \mathrm{O}_{2} \\
409.44\end{array}$ & $\begin{array}{l}70.40 / \\
70.35\end{array}$ & $\begin{array}{l}4.68 / \\
4.71\end{array}$ & $\begin{array}{l}17.10 \\
17.05\end{array}$ & $\begin{array}{c}2.24 \mathrm{~s}\left(3 \mathrm{H}, \mathrm{CH}_{3}\right) ; 2.36 \mathrm{~s},\left(3 \mathrm{H}, \mathrm{CH}_{3}-1\right) \\
6.90 \mathrm{~d},(\mathrm{~J} 7.8,1 \mathrm{H}, \mathrm{H}-4 \mathrm{Ar}) ; 7.20 \mathrm{t},(\mathrm{J} 7.8 \\
1 \mathrm{H}, \mathrm{H}-5 \mathrm{Ar}) ; 7.60-7.72 \mathrm{~m},(7 \mathrm{H}, \mathrm{H} \mathrm{Ph} \\
2,6-\mathrm{Ar} ') ; 7.83 \mathrm{dd},\left(\mathrm{J}_{6,7} 7.8, \mathrm{~J}_{7,9} 2.0,1 \mathrm{H}\right. \\
\mathrm{H}-7) ; 8.14 \mathrm{~d},\left(\mathrm{~J}_{7,9} 2.0,1 \mathrm{H}, \mathrm{H}-9\right) ; 8.32 \mathrm{~d} \\
\left(\mathrm{~J}_{6,7} 7.8,1 \mathrm{H}, \mathrm{H}-6\right) ; 10.28 \mathrm{~s},(1 \mathrm{H}, \mathrm{CONH})\end{array}$ \\
\hline $4 f$ & $\begin{array}{c}\mathrm{R} 1=\mathrm{Ph} \\
\mathrm{R} 2= \\
=3-\mathrm{MeOPh}\end{array}$ & 73 & $>300$ & $\begin{array}{c}\mathrm{C}_{24} \mathrm{H}_{19} \mathrm{~N}_{5} \mathrm{O}_{3} \\
425.44\end{array}$ & $\begin{array}{l}67.76 / \\
67.80\end{array}$ & $\begin{array}{l}4.50 / \\
4.49\end{array}$ & $\begin{array}{l}16.46 / \\
16.42\end{array}$ & $\begin{array}{c}2.36 \mathrm{~s},\left(3 \mathrm{H}, \mathrm{CH}_{3}-1\right) ; 3.70 \mathrm{~s}\left(3 \mathrm{H}, \mathrm{OCH}_{3}\right) \\
6.60 \mathrm{~d},\left(\mathrm{~J} 7.8,1 \mathrm{H}, \mathrm{H}-4 \mathrm{Ar} \mathrm{Ar}^{\prime}\right) ; 7.08-7.22 \mathrm{~m} \\
\left(2 \mathrm{H}, \mathrm{H}-5,6-\mathrm{Ar}^{\prime}\right) ; 7.35 \mathrm{~s},\left(1 \mathrm{H}, \mathrm{H}-2 \mathrm{Ar}^{\prime}\right) \\
7.42-7.56 \mathrm{~m},(5 \mathrm{H}, \mathrm{H} \mathrm{Ph}) ; 7.74 \mathrm{dd},\left(\mathrm{J}_{6,7}\right. \\
\left.\text { 7.8, } \mathrm{J}_{7,9} 2.0,1 \mathrm{H}, \mathrm{H}-7\right) ; 8.14 \mathrm{~d},\left(\mathrm{~J}_{7,9} 2.0\right. \\
1 \mathrm{H}, \mathrm{H}-9) ; 8.32 \mathrm{~d},\left(\mathrm{~J}_{6,7} 7.8,1 \mathrm{H}, \mathrm{H}-6\right) \\
10.10 \mathrm{~s},(1 \mathrm{H}, \mathrm{CONH})\end{array}$ \\
\hline $4 \mathrm{~g}$ & $\begin{array}{c}\mathrm{R} 1=4-\mathrm{FPh} \\
\mathrm{R} 2=i-\mathrm{C}_{5} \mathrm{H}_{11}\end{array}$ & 86 & $>300$ & $\begin{array}{c}\mathrm{C}_{22} \mathrm{H}_{22} \mathrm{FN}_{5} \mathrm{O}_{2} \\
407.44\end{array}$ & $\begin{array}{l}64.85 / \\
64.81\end{array}$ & $\begin{array}{l}5.44 / \\
5.46\end{array}$ & $\begin{array}{l}17.19 / \\
17.22\end{array}$ & $\begin{array}{c}0.88 \mathrm{~d},\left(\mathrm{~J} 7.0,6 \mathrm{H}, 2 \mathrm{CH}_{3}\right) ; 1.40 \mathrm{qr},(\mathrm{J} 7.0, \\
\left.2 \mathrm{H}, \mathrm{NHCH}_{2} \mathrm{CH}_{2}\right) ; 1.52-1.72 \mathrm{~m},(1 \mathrm{H}, \\
\left.\mathrm{C}{ }_{\mathrm{H}}\left(\mathrm{CH}_{3}\right)_{2}\right) ; 2.36 \mathrm{~s},\left(3 \mathrm{H}, \mathrm{CH}_{3}-1\right) ; 3.25 \mathrm{qr}, \\
\left(\mathrm{J} 7.0,2 \mathrm{H}, \mathrm{NHC}_{2} \underline{\mathrm{CH}}_{2}\right) ; 7.50 \mathrm{t}(\mathrm{J} 7.6,2 \mathrm{H}, \\
\mathrm{H}-3,5-\mathrm{Ar}) ; 7.70-7.82 \mathrm{~m},(3 \mathrm{H}, \mathrm{H} 7, \\
\mathrm{H}-2,6-\mathrm{Ar}) ; 8.00 \mathrm{~d},\left(\mathrm{~J}_{7,9} 2.0,1 \mathrm{H}, \mathrm{H}-9\right) ; \\
8.26 \mathrm{~d},\left(\mathrm{~J}_{6,7} 7.8,1 \mathrm{H}, \mathrm{H}-6\right) ; 8.55 \mathrm{t}, \\
(\mathrm{J} 7.0,1 \mathrm{H}, \mathrm{CONH})\end{array}$ \\
\hline $4 h$ & $\begin{array}{c}\mathrm{R} 1=4-\mathrm{FPh} \\
\mathrm{R} 2=4-\mathrm{MeBn}\end{array}$ & 85 & $>300$ & $\begin{array}{c}\mathrm{C}_{25} \mathrm{H}_{20} \mathrm{FN}_{5} \mathrm{O}_{2} \\
441.46\end{array}$ & $\begin{array}{l}68.02 / \\
67.97\end{array}$ & $\begin{array}{l}4.57 / \\
4.60\end{array}$ & $\begin{array}{c}15.86 / \\
15.88\end{array}$ & $\begin{array}{c}2.24 \mathrm{~s}\left(3 \mathrm{H}, \mathrm{CH}_{3}\right) ; 2.36 \mathrm{~s},\left(3 \mathrm{H}, \mathrm{CH}_{3}-1\right) \\
4.40 \mathrm{~d}\left(\mathrm{~J} 7.0,2 \mathrm{H}, \mathrm{CH}_{2}\right) ; 7.09 \mathrm{~d},(\mathrm{~J} 7.8,2 \mathrm{H} \\
3,5 \mathrm{Bn}) ; 7.19 \mathrm{~d},(\mathrm{~J} 7.8,2 \mathrm{H}, 2,6 \mathrm{Bn}) ; \\
7.50 \mathrm{t}(\mathrm{J} 7.6,2 \mathrm{H}, \mathrm{H}-3,5-\mathrm{Ar}) ; 7.70-7.82 \mathrm{~m}, \\
(3 \mathrm{H}, \mathrm{H} 7, \mathrm{H}-2,6-\mathrm{Ar}) ; 8.04 \mathrm{~d},\left(\mathrm{~J}_{7,9} 2.0\right. \\
\text { 1H, H-9); } 8.26 \mathrm{~d},\left(\mathrm{~J}_{6,7} 7.8,1 \mathrm{H}, \mathrm{H}-6\right) ; 9.20 \mathrm{t} \\
(\mathrm{J} 7.0,1 \mathrm{H}, \mathrm{CONH})\end{array}$ \\
\hline
\end{tabular}




\begin{tabular}{|c|c|c|c|c|c|c|c|c|}
\hline 1 & 2 & 3 & 4 & 5 & 6 & 7 & 8 & 9 \\
\hline $4 i$ & $\begin{array}{c}\mathrm{R} 1=4-\mathrm{FPh} \\
\mathrm{R} 2= \\
=4-\mathrm{MeOBn}\end{array}$ & 88 & $>300$ & $\begin{array}{c}\mathrm{C}_{25} \mathrm{H}_{20} \mathrm{FN}_{5} \mathrm{O}_{3} \\
457.47\end{array}$ & $\begin{array}{l}65.64 / \\
65.59\end{array}$ & $\begin{array}{l}4.41 / \\
4.39\end{array}$ & $\begin{array}{l}15.31 / \\
15.34\end{array}$ & $\begin{array}{c}2.36 \mathrm{~s},\left(3 \mathrm{H}, \mathrm{CH}_{3}-1\right) ; 3.70 \mathrm{~s}\left(3 \mathrm{H}, \mathrm{OCH}_{3}\right) ; \\
4.37 \mathrm{~d}\left(\mathrm{~J} 7.0,2 \mathrm{H}, \mathrm{CH}_{2}\right) ; 6.85 \mathrm{~d},(\mathrm{~J} 7.8 \\
2 \mathrm{H}, 3,5 \mathrm{Bn}) ; 7.22 \mathrm{~d},(\mathrm{~J} 7.8,2 \mathrm{H}, 2,6 \mathrm{Bn}) ; \\
7.50 \mathrm{t}(\mathrm{J} 7.6,2 \mathrm{H}, \mathrm{H}-3,5-\mathrm{Ar}) \\
\text { 7.70-7.82 m, }(3 \mathrm{H}, \mathrm{H} 7, \mathrm{H}-2,6-\mathrm{Ar}) ; \\
8.03 \mathrm{~d},\left(\mathrm{~J}_{7,9} 2.0,1 \mathrm{H},\right. \\
\mathrm{H}-9) ; 8.26 \mathrm{~d},\left(\mathrm{~J}_{6,7} 7.8,1 \mathrm{H}, \mathrm{H}-6\right) ; 9.18 \mathrm{t} \\
(\mathrm{J} 7.0,1 \mathrm{H}, \mathrm{CONH})\end{array}$ \\
\hline $4 \mathbf{j}$ & $\begin{array}{c}\mathrm{R} 1=4-\mathrm{FPh} \\
\mathrm{R} 2= \\
=\mathrm{Ph}-\left(\mathrm{CH}_{2}\right)_{3}\end{array}$ & 77 & $\begin{array}{l}294- \\
296\end{array}$ & $\begin{array}{c}\mathrm{C}_{26} \mathrm{H}_{22} \mathrm{FN}_{5} \mathrm{O}_{2} \\
455.49\end{array}$ & $\begin{array}{l}68.56 / \\
68.52\end{array}$ & $\begin{array}{l}4.87 / \\
4.90\end{array}$ & $\begin{array}{c}15.38 / \\
15.40\end{array}$ & $\begin{array}{c}1.80 \mathrm{qn},\left(\mathrm{J} 7.0,2 \mathrm{H}, 2-\mathrm{CH}_{2}\right) ; 2.36 \mathrm{~s}, \\
\left(3 \mathrm{H}, \mathrm{CH}_{3}-1\right) ; 2.59 \mathrm{t},\left(\mathrm{J} 7.0,2 \mathrm{H}, 3-\mathrm{CH}_{2}\right) ; \\
3.25 \mathrm{qr},\left(\mathrm{J} 7.0,2 \mathrm{H}, 1-\mathrm{CH}_{2}\right) ; \\
7.15-7.30 \mathrm{~m},(5 \mathrm{H}, \mathrm{H} \mathrm{Ph}-3) ; \\
7.50 \mathrm{t}(\mathrm{J} 7.6,2 \mathrm{H}, \mathrm{H}-3,5-\mathrm{Ar}) ; \\
\text { 7.70-7.82 m, (3H, H 7, H-2,6-Ar); } \\
8.00 \mathrm{~d},\left(\mathrm{~J}_{7,9} 2.0,1 \mathrm{H}, \mathrm{H}-9\right) ; \\
8.26 \mathrm{~d},\left(\mathrm{~J}_{6,7} 7.8,1 \mathrm{H},\right. \\
\mathrm{H}-6) ; 8.64 \mathrm{t},(\mathrm{J} 7.0,1 \mathrm{H}, \mathrm{CONH})\end{array}$ \\
\hline $4 k$ & $\begin{array}{c}\mathrm{R} 1=4-\mathrm{FPh} \\
\mathrm{R} 2=3-\mathrm{MePh}\end{array}$ & 70 & $>300$ & $\begin{array}{c}\mathrm{C}_{24} \mathrm{H}_{18} \mathrm{FN}_{5} \mathrm{O}_{2} \\
427.43\end{array}$ & $\begin{array}{l}67.44 / \\
67.39\end{array}$ & $\begin{array}{l}4.24 / \\
4.26\end{array}$ & $\begin{array}{l}16.38 / \\
16.35\end{array}$ & $\begin{array}{c}2.24 \mathrm{~s}\left(3 \mathrm{H}, \mathrm{CH}_{3}\right) ; 2.36 \mathrm{~s}, \\
\left(3 \mathrm{H}, \mathrm{CH}_{3}-1\right) ; 6.90 \mathrm{~d},(\mathrm{~J} 7.8 \\
1 \mathrm{H}, \mathrm{H}-4 \mathrm{Ar}) ; 7.20 \mathrm{t},(\mathrm{J} 7.8 \\
\left.1 \mathrm{H}, \mathrm{H}-5 \mathrm{Ar}^{\prime}\right) ; 7.44-7.70 \mathrm{~m}, \\
(4 \mathrm{H}, \mathrm{H}-3,5-\mathrm{Ar}, 2,6-\mathrm{Ar} ') ; \\
7.70-7.82 \mathrm{~m},(3 \mathrm{H}, \mathrm{H} 7, \mathrm{H}-2,6-\mathrm{Ar}) ; \\
8.14 \mathrm{~d},\left(\mathrm{~J}_{7,9} 2.0,1 \mathrm{H}, \mathrm{H}-9\right) ; \\
8.32 \mathrm{~d},\left(\mathrm{~J}_{6,7} 7.8,1 \mathrm{H}, \mathrm{H}-6\right) ; \\
10.30 \mathrm{~s},(1 \mathrm{H}, \mathrm{CONH})\end{array}$ \\
\hline 41 & $\begin{array}{c}\mathrm{R} 1=4-\mathrm{FPh} \\
\mathrm{R} 2= \\
=3-\mathrm{MeOPh}\end{array}$ & 72 & $>300$ & $\begin{array}{c}\mathrm{C}_{24} \mathrm{H}_{18} \mathrm{FN}_{5} \mathrm{O}_{3} \\
443.43\end{array}$ & $\begin{array}{l}65.01 / \\
64.97\end{array}$ & $\begin{array}{l}4.09 / \\
4.11\end{array}$ & $\begin{array}{c}15.79 / \\
15.81\end{array}$ & $\begin{array}{c}2.36 \mathrm{~s},\left(3 \mathrm{H}, \mathrm{CH}_{3}-1\right) ; 3.70 \mathrm{~s} \\
\left(3 \mathrm{H}, \mathrm{OCH}_{3}\right) ; 6.60 \mathrm{~d},(\mathrm{~J} 7.8, \\
\left.1 \mathrm{H}, \mathrm{H}-4 \mathrm{Ar}^{\prime}\right) ; 7.08-7.22 \mathrm{~m}, \\
\left(2 \mathrm{H}, \mathrm{H}-5,6-\mathrm{Ar}^{\prime}\right) ; 7.35 \mathrm{~s}, \\
\left(1 \mathrm{H}, \mathrm{H}-2 \mathrm{Ar}^{\prime}\right) ; 7.50 \mathrm{t}(\mathrm{J} 7.6, \\
2 \mathrm{H}, \mathrm{H}-3,5-\mathrm{Ar}) ; 7.70-7.82 \mathrm{~m}, \\
(3 \mathrm{H}, \mathrm{H} 7, \mathrm{H}-2,6-\mathrm{Ar}) ; 8.14 \mathrm{~d}, \\
\left(\mathrm{~J}_{7,9} 2.0,1 \mathrm{H}, \mathrm{H}-9\right) ; 8.32 \mathrm{~d}, \\
\left(\mathrm{~J}_{6,7} 7.8,1 \mathrm{H}, \mathrm{H}-6\right) ; 10.10 \mathrm{~s}, \\
(1 \mathrm{H}, \mathrm{CONH})\end{array}$ \\
\hline
\end{tabular}

Formation of the $[1,2,4]$ triazolo[4,3-a]quinazolin$5(4 H)$-ones condenced system led to shift of H-6 protons signals to $8.26-8.32 \mathrm{ppm}$, that is in good correlation with the known data [19]. Melting points were measured with a Buchi B-520 melting point apparatus. Elemental analysis was performed on Euro EA-3000 apparatus. Starting methyl 3-aryl-4-oxo-2-thioxo-1,2,3,4-tetrahydroquinazoline-7-carboxylates 1a, $\mathbf{b}$ have been obtained from commercial producer.

The virtual screening for biological activity of virtual library of studied substances was performed by the PASS Online web-resource, which contains information about structure and biological activity more than 300000 organic compounds [20-22]. Computer prediction of the biological activity spectrum of virtual library of 4-aryl-5oxo-4,5-dihydro[1,2,4]triazolo[4,3- $a$ ]quinazoline-8-carboxamides 4a-l was performed with probability of demonsration of specific type of therapeutic action exceeding $50 \%(\mathrm{~Pa}>0,500)$.

Analysis of the computer prediction results for the virual library of 4-aryl-5-oxo-4,5-dihydro[1,2,4]tria-zo- lo[4,3-a]quinazoline-8-carboxamides by PASS software showed the possibility of searching substances possessing potential antineurotic activity, and perspective for male reproductive and erectile disfunction treatment among these compounds and allowed to generate the library of the most perspective compounds 4a-l for further investigations (Table 2). However, the probability of antiasthmatic and antiallergic activity is diminished compared to previously described library of having no amide group compounds [23].

Prediction of acute toxicity of compounds $4 \mathbf{a}-\mathbf{l}$ for different routes of administration (oral, subcutaneous, intravenous, intraperitoneal) has been carried out by GUSAR software [24, 25]. The training set of program was developed based on SYMYX MDL Toxicity Database, which contains information about acute toxicity more than 10000 chemical structures. Obtained data of acute toxicity of the studied compounds are presented by $\mathrm{LD}_{50}$ value $(\log 10(\mathrm{mmol} / \mathrm{kg})$ and $\mathrm{mg} / \mathrm{kg})$ and the toxicity class according to the OECD classification project of chemical substance by acute toxicity values [24, 26]. 
Prediction of the biological activity spectrum of

Table 2

4-aryl-5-oxo-4,5-dihydro[1,2,4]triazolo[4,3-a]quinazoline-8-carboxamides 4a-l [23]

\begin{tabular}{|c|c|c|c|c|c|c|c|c|c|c|c|c|}
\hline \multirow{3}{*}{$\begin{array}{l}\text { Biological } \\
\text { activity }\end{array}$} & \multicolumn{2}{|c|}{$4 \mathbf{a}$} & \multicolumn{2}{|c|}{$4 b$} & \multicolumn{2}{|c|}{$4 c$} & \multicolumn{2}{|c|}{$4 d$} & \multicolumn{2}{|c|}{$4 e$} & \multicolumn{2}{|c|}{$4 f$} \\
\hline & & & & & & & & & & & & \\
\hline & $\mathrm{Pa}$ & $\mathrm{Pi}$ & $\mathrm{Pa}$ & $\mathrm{Pi}$ & $\mathrm{Pa}$ & $\mathrm{Pi}$ & $\mathrm{Pa}$ & $\mathrm{Pi}$ & $\mathrm{Pa}$ & $\mathrm{Pi}$ & $\mathrm{Pa}$ & $\mathrm{Pi}$ \\
\hline 1 & 2 & 3 & 4 & 5 & 6 & 7 & 8 & 9 & 10 & 11 & 12 & 13 \\
\hline Antineurotic & 0.569 & 0.083 & 0.534 & 0.096 & 0.605 & 0.070 & 0.730 & 0.032 & 0.306 & 0.241 & 0.456 & 0.131 \\
\hline $\begin{array}{c}\text { Erectile } \\
\text { dysfunction } \\
\text { treatment }\end{array}$ & 0.786 & 0.003 & 0.802 & 0.003 & 0.774 & 0.003 & 0.770 & 0.004 & 0.775 & 0.003 & 0.748 & 0.004 \\
\hline $\begin{array}{l}\text { Male } \\
\text { reproductive } \\
\text { disfunction } \\
\text { treatment }\end{array}$ & 0.733 & 0.004 & 0.746 & 0.004 & 0.722 & 0.004 & 0.736 & 0.004 & 0.742 & 0.004 & 0.715 & 0.004 \\
\hline $\begin{array}{l}\text { Phobic } \\
\text { disorders } \\
\text { treatment }\end{array}$ & 0.660 & 0.095 & - & - & - & - & 0.346 & 0.279 & - & - & - & - \\
\hline $\begin{array}{l}\text { Fibrinogen } \\
\text { receptor } \\
\text { antagonist }\end{array}$ & 0.533 & 0.033 & 0.424 & 0.073 & 0.380 & 0.092 & 0.499 & 0.044 & - & - & - & - \\
\hline $\begin{array}{l}\text { Tumour necro- } \\
\text { sis factor alpha } \\
\text { release } \\
\text { inhibitor }\end{array}$ & 0.493 & 0.009 & 0.469 & 0.009 & 0.477 & 0.009 & 0.456 & 0.010 & 0.540 & 0.007 & 0.524 & 0.007 \\
\hline $\begin{array}{c}\text { Thiol protease } \\
\text { inhibitor }\end{array}$ & 0.451 & 0.016 & - & - & - & - & - & - & - & - & - & - \\
\hline Anticonvulsant & 0.440 & 0.050 & 0.345 & 0.092 & - & - & 0.314 & 0.112 & - & - & - & - \\
\hline $\begin{array}{c}\text { Dysmenorrhea } \\
\text { treatment }\end{array}$ & 0.392 & 0.002 & 0.445 & 0.002 & 0.392 & 0.002 & 0.410 & 0.002 & 0.480 & 0.002 & 0.410 & 0.002 \\
\hline Antiasthmatic & 0.426 & 0.041 & 0.567 & 0.019 & 0.579 & 0.018 & 0.541 & 0.022 & 0.611 & 0.014 & 0.615 & 0.014 \\
\hline $\begin{array}{l}\text { Interleukin } \\
2 \text { antagonist }\end{array}$ & 0.377 & 0.004 & 0.416 & 0.004 & 0.361 & 0.004 & 0.383 & 0.004 & 0.566 & 0.003 & 0.488 & 0.003 \\
\hline $\begin{array}{l}\text { Platelet } \\
\text { aggregation } \\
\text { inhibitor }\end{array}$ & 0.370 & 0.027 & 0.388 & 0.023 & 0.399 & 0.021 & 0.416 & 0.018 & 0.434 & 0.015 & 0.442 & 0.014 \\
\hline Antiallergic & 0.390 & 0.056 & 0.501 & 0.028 & 0.528 & 0.024 & 0.495 & 0.029 & 0.581 & 0.017 & 0.591 & 0.016 \\
\hline $\begin{array}{l}\text { HIV } \\
\text { attachment } \\
\text { inhibitor }\end{array}$ & 0.321 & 0.034 & 0.387 & 0.014 & 0.375 & 0.017 & 0.305 & 0.042 & 0.320 & 0.035 & 0.315 & 0.037 \\
\hline $\begin{array}{c}\text { Interferon } \\
\text { alpha agonist }\end{array}$ & 0.335 & 0.049 & - & - & - & - & - & - & - & - & - & - \\
\hline $\begin{array}{l}\text { CYP2C8 } \\
\text { inhibitor }\end{array}$ & 0.382 & 0.138 & - & - & - & - & - & - & 0.312 & 0.214 & - & - \\
\hline $\begin{array}{l}\text { Kidney } \\
\text { function } \\
\text { stimulant }\end{array}$ & - & - & 0.382 & 0.182 & - & - & 0.321 & 0.232 & - & - & - & - \\
\hline $\begin{array}{l}\text { Gluconate 2- } \\
\text { dehydrogenase } \\
\text { (acceptor) } \\
\text { inhibitor }\end{array}$ & - & - & - & - & 0.398 & 0.300 & - & - & - & - & - & - \\
\hline
\end{tabular}




\begin{tabular}{|c|c|c|c|c|c|c|c|c|c|c|c|c|}
\hline 1 & 2 & 3 & 4 & 5 & 6 & 7 & 8 & 9 & 10 & 11 & 12 & 13 \\
\hline $\begin{array}{l}\text { CYP2H } \\
\text { substrate }\end{array}$ & - & - & - & - & 0.360 & 0.270 & - & - & - & - & - & - \\
\hline $\begin{array}{l}\text { Platelet de- } \\
\text { rived growth } \\
\text { factor receptor } \\
\text { kinase inhibi- } \\
\text { tor }\end{array}$ & - & - & - & - & - & - & - & - & 0.480 & 0.036 & 0.389 & 0.079 \\
\hline $\begin{array}{l}\text { GABA } \\
\text { receptor } \\
\text { agonist }\end{array}$ & - & - & - & - & - & - & - & - & - & - & - & - \\
\hline $\begin{array}{c}\text { Antiinflammat } \\
\text { ory }\end{array}$ & - & - & - & - & - & - & - & - & - & - & - & - \\
\hline \multirow{3}{*}{$\begin{array}{l}\text { Biological } \\
\text { activity }\end{array}$} & \multicolumn{2}{|c|}{$4 g$} & \multicolumn{2}{|c|}{$4 h$} & \multicolumn{2}{|c|}{$4 \mathbf{i}$} & \multicolumn{2}{|c|}{$4 \mathbf{j}$} & \multicolumn{2}{|c|}{$4 k$} & \multicolumn{2}{|c|}{41} \\
\hline & & & & & & & & & & & & \\
\hline & $\mathrm{Pa}$ & $\mathrm{Pi}$ & $\mathrm{Pa}$ & $\mathrm{Pi}$ & $\mathrm{Pa}$ & $\mathrm{Pi}$ & $\mathrm{Pa}$ & $\mathrm{Pi}$ & $\mathrm{Pa}$ & $\mathrm{Pi}$ & $\mathrm{Pa}$ & $\mathrm{Pi}$ \\
\hline Antineurotic & 0.690 & 0.043 & 0.662 & 0.052 & 0.716 & 0.036 & 0.803 & 0.016 & 0.419 & 0.151 & 0.592 & 0.074 \\
\hline $\begin{array}{l}\text { Erectile } \\
\text { dysfunction } \\
\text { treatment }\end{array}$ & 0.763 & 0.004 & 0.777 & 0.003 & 0.751 & 0.004 & 0.736 & 0.004 & 0.740 & 0.004 & 0.713 & 0.004 \\
\hline $\begin{array}{c}\text { Male } \\
\text { reproductive } \\
\text { disfunction } \\
\text { treatment }\end{array}$ & 0.713 & 0.004 & 0.726 & 0.004 & 0.703 & 0.004 & 0.706 & 0.004 & 0.710 & 0.004 & 0.687 & 0.004 \\
\hline $\begin{array}{l}\text { Phobic } \\
\text { disorders } \\
\text { treatment }\end{array}$ & 0.476 & 0.187 & - & - & - & - & - & - & - & - & - & - \\
\hline $\begin{array}{l}\text { Fibrinogen } \\
\text { receptor } \\
\text { antagonist }\end{array}$ & 0.353 & 0.106 & - & - & - & - & - & - & - & - & - & - \\
\hline $\begin{array}{c}\text { Tumour necro- } \\
\text { sis factor alpha } \\
\text { release } \\
\text { inhibitor }\end{array}$ & 0.512 & 0.008 & 0.493 & 0.009 & 0.497 & 0.008 & 0.468 & 0.010 & 0.542 & 0.006 & 0.527 & 0.007 \\
\hline $\begin{array}{c}\text { Thiol protease } \\
\text { inhibitor }\end{array}$ & 0.393 & 0.024 & - & - & - & - & 0.316 & 0.111 & - & - & - & - \\
\hline $\begin{array}{c}\text { Anti- } \\
\text { convulsant }\end{array}$ & 0.431 & 0.053 & 0.338 & 0.097 & - & - & - & - & - & - & - & - \\
\hline $\begin{array}{c}\text { Dysmenorrhea } \\
\text { treatment }\end{array}$ & 0.375 & 0.002 & 0.414 & 0.002 & 0.376 & 0.002 & 0.385 & 0.002 & 0.432 & 0.002 & 0.385 & 0.002 \\
\hline Antiasthmatic & 0.409 & 0.045 & 0.541 & 0.022 & 0.557 & 0.020 & 0.510 & 0.026 & 0.575 & 0.018 & 0.583 & 0.017 \\
\hline $\begin{array}{l}\text { Interleukin } \\
2 \text { antagonist }\end{array}$ & 0.362 & 0.004 & 0.392 & 0.004 & 0.347 & 0.004 & 0.361 & 0.004 & 0.522 & 0.003 & 0.448 & 0.003 \\
\hline $\begin{array}{c}\text { Platelet aggre- } \\
\text { gation } \\
\text { inhibitor }\end{array}$ & 0.332 & 0.038 & 0.343 & 0.034 & 0.359 & 0.029 & 0.369 & 0.027 & 0.383 & 0.024 & 0.396 & 0.021 \\
\hline Antiallergic & 0.383 & 0.058 & 0.487 & 0.030 & 0.513 & 0.026 & 0.477 & 0.033 & 0.554 & 0.020 & 0.566 & 0.019 \\
\hline $\begin{array}{c}\text { HIV } \\
\text { attachment } \\
\text { inhibitor }\end{array}$ & 0.337 & 0.028 & 0.397 & 0.012 & 0.385 & 0.014 & 0.325 & 0.033 & 0.339 & 0.027 & 0.333 & 0.029 \\
\hline
\end{tabular}




\begin{tabular}{|c|c|c|c|c|c|c|c|c|c|c|c|c|}
\hline 1 & 2 & 3 & 4 & 5 & 6 & 7 & 8 & 9 & 10 & 11 & 12 & 13 \\
\hline $\begin{array}{c}\text { Interferon } \\
\text { alpha agonist }\end{array}$ & - & - & - & - & - & - & - & - & - & - & - & - \\
\hline $\begin{array}{l}\text { CYP2C8 } \\
\text { inhibitor }\end{array}$ & 0.331 & 0.192 & - & - & - & - & - & - & - & - & - & - \\
\hline $\begin{array}{l}\text { Kidney } \\
\text { function } \\
\text { stimulant }\end{array}$ & - & - & - & - & - & - & - & - & - & - & - & - \\
\hline $\begin{array}{l}\text { Gluconate } \\
\text { 2-dehydro- } \\
\text { genase (accep- } \\
\text { tor) inhibitor }\end{array}$ & - & - & - & - & - & - & - & - & - & - & - & - \\
\hline $\begin{array}{l}\text { CYP2H } \\
\text { substrate }\end{array}$ & - & - & - & - & - & - & - & - & - & - & - & - \\
\hline $\begin{array}{l}\text { Platelet derived } \\
\text { growth factor } \\
\text { receptor kinase } \\
\text { inhibitor }\end{array}$ & - & - & 0.348 & 0.112 & 0.321 & 0.139 & 0.345 & 0.114 & 0.510 & 0.028 & 0.426 & 0.058 \\
\hline $\begin{array}{l}\text { GABA } \\
\text { receptor } \\
\text { agonist }\end{array}$ & 0.317 & 0.025 & 0.313 & 0.026 & - & - & - & - & - & - & - & - \\
\hline $\begin{array}{c}\text { Antiinflammat } \\
\text { ory }\end{array}$ & - & - & - & - & 0.301 & 0.159 & - & - & 0.306 & 0.155 & 0.308 & 0.153 \\
\hline
\end{tabular}

Results of investigation in silico by GUSAR software gave the possbility to predict acute toxicity values for different routes of administration of 4-aryl5-oxo-4,5-dihydro[1,2,4]triazolo[4,3- $a]$ quinazoline-8-carboxamides 4a-l (Table 3 ). The values $\mathrm{LD}_{50}$ by oral administration were between 511 to $1795 \mathrm{mg} / \mathrm{kg}$, by subcutaneous -1057 to $3045 \mathrm{mg} / \mathrm{kg}$. The values $\mathrm{LD}_{50}$ by intravenous administration were between 94 to $215 \mathrm{mg} / \mathrm{kg}$, and by intraperitoneal - were between 315 to $769 \mathrm{mg} / \mathrm{kg}$. The data obtained indicate that compounds $\mathbf{4 a - 1}$ are slightly toxic (class 4 ) or practically nontoxic (class 5) [24, 26].

Table 3

The values of acute toxicity of 4-aryl-5-oxo-4,5-dihydro[1,2,4]triazolo[4,3-a]quinazoline-8-carboxamides 4a-I [26]

\begin{tabular}{|c|c|c|c|c|c|}
\hline \multirow{2}{*}{$\begin{array}{l}\text { Compound } \\
\text { code }\end{array}$} & \multirow[b]{2}{*}{$\mathrm{R}$} & \multicolumn{4}{|c|}{$\mathrm{LD}_{50}, \mathrm{mg} / \mathrm{kg}$} \\
\hline & & $\begin{array}{c}\text { Oral } \\
\text { administration } \\
\end{array}$ & $\begin{array}{r}\text { Subcutaneous } \\
\text { administration }\end{array}$ & $\begin{array}{c}\text { Intravenous admin- } \\
\text { istration }\end{array}$ & $\begin{array}{l}\text { Intraperitoneal } \\
\text { administration }\end{array}$ \\
\hline $4 a$ & $\mathrm{R} 1=\mathrm{Ph} ; \mathrm{R} 2=i-\mathrm{C}_{5} \mathrm{H}_{11}$ & 633 & 1361 & 94 & 425 \\
\hline $4 b$ & $\mathrm{R} 1=\mathrm{Ph} ; \mathrm{R} 2=4-\mathrm{MeBn}$ & 1795 & 1798 & 143 & 497 \\
\hline $4 \mathrm{c}$ & $\mathrm{R} 1=\mathrm{Ph} ; \mathrm{R} 2=4-\mathrm{MeOBn}$ & 512 & 1057 & 177 & 654 \\
\hline $4 d$ & $\mathrm{R} 1=\mathrm{Ph} ; \mathrm{R} 2=\mathrm{Ph}\left(\mathrm{CH}_{2}\right)_{3}$ & 998 & 1469 & 121 & 456. \\
\hline $4 \mathrm{e}$ & $\mathrm{R} 1=\mathrm{Ph} ; \mathrm{R} 2=3-\mathrm{MePh}$ & 995 & 1695 & 210 & 507 \\
\hline $4 f$ & $\mathrm{R} 1=\mathrm{Ph} ; \mathrm{R} 2=3-\mathrm{MeOPh}$ & 970 & 1840 & 202 & 672 \\
\hline $4 \mathrm{~g}$ & $\mathrm{R} 1=4-\mathrm{FPh} ; \mathrm{R} 2=i-\mathrm{C}_{5} \mathrm{H}_{11}$ & 693 & 1393 & 115 & 455 \\
\hline $4 \mathrm{~h}$ & $\mathrm{R} 1=4-\mathrm{FPh} ; \mathrm{R} 2=4-\mathrm{MeBn}$ & 1119 & 1686 & 216 & 651 \\
\hline $4 \mathrm{i}$ & $\mathrm{R} 1=4-\mathrm{FPh} ; \mathrm{R} 2=4-\mathrm{MeOBn}$ & 603 & 3543 & 148 & 341 \\
\hline $4 j$ & $\mathrm{R} 1=4-\mathrm{FPh} ; \mathrm{R} 2=\mathrm{Ph}\left(\mathrm{CH}_{2}\right)_{3}$ & 805 & 1760 & 189 & 316 \\
\hline $4 \mathrm{k}$ & $\mathrm{R} 1=4-\mathrm{FPh} ; \mathrm{R} 2=3-\mathrm{MePh}$ & 1668 & 2045 & 186 & 769 \\
\hline 41 & $\mathrm{R} 1=4-\mathrm{FPh} ; \mathrm{R} 2=3-\mathrm{MeOPh}$ & 589 & 1763 & 187 & 353 \\
\hline
\end{tabular}

\section{Conclusions}

According to the result of computer prediction of the biological activity spectrum and acute toxicity of 4 aryl-5-oxo-4,5-dihydro[1,2,4]triazolo[4,3-a]quinazoline8 -carboxamides the selection of slightly toxic or nontoxic substances with the potential antineurotic activi- ty, and perspective for male reproductive and erectile dysfunction treatment has been performed.

\section{References}

1. Alagarsamy V. Synthesis and pharmacological investigation of novel 1-substituted-4-phenyl-1,2,4-triazolo[4,3- 
a]quinazolin-5(4H)-ones as a new class of H1-antihistaminic agents [Text] / V. Alagarsamy, R. Giridhar, M. R. Yadav // Bioorganic \& Medicinal Chemistry Letters. - 2005. - Vol. 15, Issue 7. - P. 1877-1880. doi: 10.1016/j.bmcl.2005.02.016

2. Alagarsamy, V. Synthesis and pharmacological investigation of novel 1-substituted-4-(4-substituted phenyl)- $4 \mathrm{H}$ $[1,2,4]$ triazolo[4,3-a]quinazolin-5-ones as a new class of H1-antihistamine agents [Text] / V. Alagarsamy, R. Giridhar, M. R. Yadav // Journal of Pharmacy and Pharmacology. - 2006. Vol. 58, Issue 9. - P. 1249-1255. doi: 10.1211/jpp.58.9.0012

3. Alagarsamy, V. 4-Cyclohexyl-1-substituted-4H-[1,2,4]triazolo[4,3- $a$ ]quinazolin-5-ones: Novel Class of H1-antihistaminic Agents [Text] / V. Alagarsamy, S. Meena, K. V. Ramaseshu // Chemical Biology \& Drug Design. - 2007. - Vol. 70, Issue 2. - P. 158-163. doi: 10.1111/j.1747-0285.2007.00544.X

4. Alagarsamy, V. Synthesis and pharmacological investigation of novel 4-benzyl-1-substituted-4H-[1,2,4]triazolo [4,3-a]quinazolin-5-ones as new class of $\mathrm{H} 1$-antihistaminic agents [Text] / V. Alagarsamy, V. R. Solomon, M. Murugan // Bioorganic \& Medicinal Chemistry. - 2007. - Vol. 45, Issue 12. - P. 4009-4015. doi: 10.1016/j.bmc.2007.04.001

5. Alagarsamy, V. Synthesis and pharmacological investigation of novel 4-(2-methylphenyl)-1-substituted-4H-[1,2,4] triazolo[4,3-a]quinazolin-5-ones as new class of H1-antihistaminic agents [Text] / V. Alagarsamy, M. Rupeshkumar, K. Kavitha, S. Meena, D. Shankar, A. A. Siddiqui, R. Rajesh // European Journal of Medicinal Chemistry. - 2008. - Vol. 43, Issue 11. - P. 2331-2337. doi: 10.1016/j.ejmech.2007.10.001

6. Alagarsamy, V. Synthesis and pharmacological investigation of novel 4-(3-ethylphenyl)-1-substituted-4H-[1,2,4] triazolo[4,3-a]quinazolin-5-ones as a new class of H1-antihistaminic agents [Text] / V. Alagarsamy, K. Kavitha, M. Rupeshkumar, V. R. Solomon, J. Kumar, D. Kumar, H. Sharma // Acta Pharmaceutica. - 2009. - Vol. 59, Issue 1. - P. 97-106. doi: 10.2478/v10007-009-0003-1

7. Alagarsamy, V. 4-(3-Methoxyphenyl)-1-substituted$4 H$-[1,2,4]triazolo[4,3-a]quinazolin-5-ones: new class of H1antihistaminic agents [Text] / V. Alagarsamy, H. K. Sharma, P. Parthiban et. al. // Pharmazie. - 2009. - Vol. 64. - P. 5-9.

8. Gobinath, M. Design, synthesis and H1-antihistaminic activity of novel 1-substituted-4-(3-chlorophenyl)-[1,2,4] triazolo[4,3-a]quinazolin-5(4H)-ones [Text] / M. Gobinatha, N. Subramanianb, V. Alagarsamy // Journal of Saudi Chemical Society. - 2015. -Vol. 19, Issue 3. - P. 282-286. doi: 10.1016/ j.jscs.2012.02.006

9. Gobinath, M. Synthesis of 1-substituted-4-(pyridin-4yl) $[1,2,4]$ triazolo[4,3-a]quinazolin-5(4H)-ones as a new class of H1-antihistaminic agents [Text] / M. Gobinath, N. Subramanian, V. Alagarsamy, Nivedhitha S., Solomon V. Raja // Tropical Journal of Pharmaceutical Research. - 2015. - Vol. 14, Issue 2. P. 271-277. doi: 10.4314/tjpr.v14i2.12

10. Abdel Gawad, N. M. Design, synthesis, and anticonvulsant activity of novel quinazolinone analogues [Text] / N. M. Abdel Gawad, H. H. Georgey, R. M. Youssef, N. A. El Sayed // Medicinal Chemistry Research. - 2011. - Vol. 20, Issue 8. - P. 1280-1286. doi: 10.1007/s00044-010-9465-4

11. Alagarsamy, V. AntiHIV, antibacterial and antifungal activities of some novel 1,4-disubstituted-1,2,4-triazolo[4,3a]quinazolin-5(4H)-ones [Text] / V. Alagarsamy, R. Giridhar, M. R. Yadav, R. Revathi, K. Ruckmani, E. De Clercq // Indian Journal of Pharmaceutical Sciences. - 2006. - Vol. 68, Issue 4. - P. 532-535. doi: 10.4103/0250-474x.27840

12. Deshmukh, M. B. Synthesis and antimicrobial screening of pyrazolo-3-aryl quinazolin-4(3H)ones [Text] / M. B. Deshmukh, S. Patil, S. S. Pati, S. D. Jadhav // Indian Journal of Pharmaceutical Sciences. - 2010. - Vol. 72, Issue 4. - P. 500504. doi: 10.4103/0250-474x.73934

13. Gobinath, M. Anticancer and antitubercular study of some 1,4-disubstituted[1,2,4]-triazolo[4,3-a]quinazolin-5(4H)-on- es [Text] / M. Gobinath, N. Subramanian, V. Alagarsamy // International Journal of Research in Pharmaceutical Sciences. 2012. - Vol. 3, Issue 1. - P. 146-149.

14. Patent 2792938. Nouvelles 1-aminotriazolo[4,3a]quinazoline-5-ones inhibitrices de phosphodiesterases IV [Text] / Gaudilliere B., Lavalette R., Andrianjara C. - France, 2000.

15. Elansary, A. K. Design, synthesis and in vitro PDE4 inhibition activity of certain quinazolinone derivatives for treatment of asthma [Text] / A. K. Elansary, H. H. Kadry, E. M. Ahmed, A. S. M. Sonousi // Medicinal Chemistry Research. 2012. - Vol. 21, Issue 11. - P. 3327-3335. doi: 10.1007/s00044011-9846-3

16. Danylchenko, S. Yu. Synthesis, computer prediction of biologi-cal activity and acute toxicity of 1-Ar-4-R-[1,2,4] triazolo[4,3-a]quinazolin-5(4H)-ones [Text] / S. Yu. Danyl-chenko, O. G. Drushlyak, S. M. Kovalenko // Journal of Organic and Pharmaceutical Chemistry. - 2015. - Vol. 13, Issue 3 (51). - P. 38-45.

17. Danylchenko, S. Yu. Formation of 1-methyl $[1,2,4]$ triazolo[4,3-a]quinazolin-5(4H)-ones by reaction of 2-hydrazinoquinazolin-4(3H)-ones with acetylacetone [Text] / S. Yu. Danylchenko, O. G. Drushlyak, S. S. Kovalenko, S. M. Kovalenko // Heterocyclic Communications. - 2015. - Vol. 21, Issue 4. P. 195-197. doi: 10.1515/hc-2015-0104

18. Danilchenko, S. Yu. Synthesis of substituted 2-hydrazinoquinazolin-4-ones as intermediates for heterocyclic compounds synthesis [Text] / S. Yu. Danilchenko, O. G. Dru-shlyak, S. M. Kovalenko // Journal of Organic and Pharmaceutical Chemistry. - 2014. - Vol. 12, Issue 3. - P. 66-73.

19. Saleh, M. A. Synthesis of aldehydo sugar (4-oxoquinazolin-2-yl)hydrazones and their transformation into 1-(alditol-1-yl)-1,2,4-triazolo[4,3-a]quinazolin-5(4H)-ones [Text] / M. A. Saleh, M. F. Abdel-Megeed, M. A. Abdo, A. M. Shkor // Journal of Heterocyclic Chemistry. - 2003. - Vol. 40, Issue 1. P. 85-92. doi: 0.1002/jhet.5570400111

20. Filimonov, D. A. Prediction of the Biological Activity Spectra of Organic Compounds Using the Pass Online Web Resource [Text] / D. A. Filimonov, A. A. Lagunin, T. A. Gloriozova, A. V. Rudik, D. S. Druzhilovskii, P. V. Pogodin, V. V. Poroikov // Chemistry of Heterocyclic Compounds. - 2014. Vol. 50, Issue 3. - P. 444-457. doi: 10.1007/s10593-014-1496-1

21. Filimonov, D. A. Prediction of biological activity of organic compounds [Text] / D. A. Filimonov, V. V. Poroikov // Rosiiskii himicheskii zhurnal. - 2006. - Vol. 2. - P. 66-75.

22. Filimonov, D. A. In Chemoinformatics Approaches to Virtual Screening [Text] / D. A. Filimonov, V. V. Poroikov; A. Varnek., A. Tropsha (Eds.). - Cambridge (UK): RSC Publishing, 2008. - P. 182-216.

23. Get more information about biological potential of your compounds [Electronic resource]. - Available at: http:// www.pharmaexpert.ru/passonline

24. Berezovskaya, I. V. Classification of the chemical parameters on acute toxicity of parenteral routes of administration [Text] / I. V. Berezovskaya // Khimiko-Farmatsevticheskii Zhurnal. - 2003. - Vol. 37, Issue 3. - P. 32-34.

25. Lagunin, A. QSAR Modelling of Rat Acute Toxicity on the Basis of PASS Prediction [Text] / A. Lagunin, A. Zakharov, D. Filimonov, V. Poroikov // Molecular Informatics. - 2011. - Vol. 30, Issue 2-3. - P. 241-250. doi: 10.1002/ minf.201000151

26. GUSAR Online [Electronic resource]. - Available at: http://pharmaexpert.ru/GUSAR/acutoxpredict.html

\section{References}

1. Alagarsamy, V., Giridhar, R., Yadav, M. R. (2005). Synthesis and pharmacological investigation of novel 1-substituted-4-phenyl-1,2,4-triazolo[4,3-a]quinazolin-5(4H)-ones as a new class of H1-antihistaminic agents. Bioorganic \& Medicinal Chemistry Letters, 15 (7), 1877-1880. doi: 10.1016/j.bmcl. 2005.02.016 
2. Alagarsamy, V., Giridhar, R., Yadav, M. R. (2006). Synthesis and pharmacological investigation of novel 1substituted-4-(4-substituted phenyl)-4 H -[1,2,4]triazolo[4,3- a ]quinazolin-5-ones as a new class of $\mathrm{H} 1$-antihistamine agents . Journal of Pharmacy and Pharmacology, 58 (9), 1249-1255. doi: 10.1211/jpp.58.9.0012

3. Alagarsamy, V., Meena, S., Ramaseshu, K. V., Solomon, V. R., Kumar, T. D. A. (2007). 4-Cyclohexyl-1-substituted4H-[1,2,4]triazolo [4,3-a] quinazolin-5-ones: Novel Class of H 1 antihistaminic Agents . Chemical Biology \& Drug Design, 70 (2), 158-163. doi: 10.1111/j.1747-0285.2007.00544.x

4. Alagarsamy, V., Solomon, V. R., Murugan, M. (2007). Synthesis and pharmacological investigation of novel 4-benzyl-1substituted-4H-[1,2,4]triazolo[4,3-a]quinazolin-5-ones as new class of H1-antihistaminic agents. Bioorganic \& Medicinal Chemistry, 15 (12), 4009-4015. doi: 10.1016/j.bmc.2007.04.001

5. Alagarsamy, V., Rupeshkumar, M., Kavitha, K., Meena, S., Shankar, D., Siddiqui, A. A., Rajesh, R. (2008). Synthesis and pharmacological investigation of novel 4-(2methylphenyl)-1-substituted-4H-[1,2,4]triazolo[4,3-a]quinazolin5 -ones as new class of H1-antihistaminic agents. European Journal of Medicinal Chemistry, 43 (11), 2331-2337. doi: 10.1016/ j.ejmech.2007.10.001

6. Alagarsamy, V., Kavitha, K., Rupeshkumar, M., Solomon, V., Kumar, J., Kumar, D., Sharma, H. (2009). Synthesis and pharmacological investigation of novel 4-(3-ethylphenyl)-1substituted-4H-[1,2,4]triazolo[4,3-a] quinazolin-5-ones as a new class of H1-antihistaminic agents. Acta Pharmaceutica, 59 (1), 97-106 doi: 10.2478/v10007-009-0003-1

7. Alagarsamy, V., Sharma, H. K., Parthiban, P. et. al. (2009). 4-(3-Methoxyphenyl)-1-substituted-4H-[1,2,4]triazolo[4,3a]quinazolin-5-ones: new class of H1-antihistaminic agents. Pharmazie, 64, 5-9.

8. Gobinath, M., Subramanian, N., Alagarsamy, V. (2015). Design, synthesis and H1-antihistaminic activity of novel 1-substituted-4-(3-chlorophenyl)-[1,2,4] triazolo [4,3-a] quinazolin-5(4H)-ones. Journal of Saudi Chemical Society, 19 (3), 282-286. doi: 10.1016/j.jscs.2012.02.006

9. Gobinath, M., Subramanian, N., Alagarsamy, V., Nivedhitha, S., Solomon, V. (2015). Synthesis of 1-Substituted4-(Pyridin-4-yl) $[1,2,4]$ Triazolo [4,3-a] Quinazolin-5(4H)-ones as a New Class of H1- Antihistaminic Agents. Tropical Journal of Pharmaceutical Research, 14 (2), 271-277. doi: 10.4314/tjpr.v14i2.12

10. Abdel Gawad, N. M., Georgey, H. H., Youssef, R. M., El Sayed, N. A. (2010). Design, synthesis, and anticonvulsant activity of novel quinazolinone analogues. Medicinal Chemistry Research, 20 (8), 1280-1286. doi: 10.1007/s00044-010-9465-4

11. Alagarsamy, V., Giridhar, R., Yadav, M., Revathi, R., Ruckmani, K., De Clercq, E. (2006). AntiHIV, antibacterial and antifungal activities of some novel 1,4-disubstituted-1,2,4triazolo[4,3-a] quinazolin-5(4 h )-ones. Indian J Pharm Sci, 68 (4), 532-535. doi: 10.4103/0250-474x.27840

12. Patil, S., Patil, S., Jadhav, S., Deshmukh, M. (2010). Synthesis and antimicrobial screening of pyrazolo-3-aryl quinazolin-4(3h)ones. Indian Journal of Pharmaceutical Sciences, 72 (4), 500-504. doi: 10.4103/0250-474x.73934
13. Gobinath, M., Subramanian, N., Alagarsamy, V. (2012), Anticancer and antitubercular study of some 1,4disubstituted[1,2,4]-triazolo[4,3-a]quinazolin-5(4H)-ones. International Journal of Research in Pharmaceutical Sciences, 3 (1), 146-149.

14. Gaudilliere, B., Lavalette, R., Andrianjara, C. (2000). Patent 2792938. Nouvelles 1-aminotriazolo[4,3-a]quinazoline-5ones inhibitrices de phosphodiesterases IV. France.

15. Elansary, A. K., Kadry, H. H., Ahmed, E. M.\& Sonousi, A. S. M. (2011). Design, synthesis and in vitro PDE4 inhibition activity of certain quinazolinone derivatives for treatment of asthma. Medicinal Chemistry Research, 21 (11), $3327-$ 3335. doi: 10.1007/s00044-011-9846-3

16. Danylchenko, S. Y., Drushlyak, O. G., Kovalenko, S. S., Kovalenko, S. M. (2015). Formation of 1-methyl[1,2,4] triazolo[4,3-a] quinazolin-5(4H)-ones by reaction of 2-hydrazinoquinazolin-4(3H)-ones with acetylacetone. Heterocyclic Communications, 21 (4), 195-197. doi: 10.1515/hc-2015-0104

17. Danylchenko, S. Yu., Drushlyak, O. G., Kovalenko, S. S., Kovalenko, S. M. (2015). Formation of 1-methyl $[1,2,4]$ triazolo[4,3-a]quinazolin-5(4H)-ones by reaction of 2hydrazinoquinazolin-4(3H)-ones with acetylacetone. Heterocyclic Communications, 21 (4), 195-197.

18. Danilchenko, S. Yu., Drushlyak, O. G., Kovalen ko, S. M. (2014). Synthesis of substituted 2-hydrazinoquinazolin4-ones as intermediates for heterocyclic compounds synthesis. Journal of Organic and Pharmaceutical Chemistry, 12 (3), 66-73.

19. Saleh, M. A., Abdel-Megeed, M. F., Abdo, M. A., Shkor, A.-B. M. (2003). Synthesis of Aldehydo Sugar (4oxoquinazolin-2-yl)hydrazones and their transformation into 1(alditol-1-yl)-1,2,4-triazolo-[4,3-a]quinazolin-5(4H)-ones. Journal of Heterocyclic Chemistry, 40 (1), 85-92. doi: 10.1002/ jhet.5570400111

20. Filimonov, D. A., Lagunin, A. A., Gloriozova, T. A., Rudik, A. V., Druzhilovskii, D. S., Pogodin, P. V., Poroikov, V. V. (2014). Prediction of the Biological Activity Spectra of Organic Compounds Using the Pass Online Web Resource. Chemistry of Heterocyclic Compounds, 50(3), 444-457. doi: 10.1007/s10593-014-1496-1

21. Filimonov, D. A., Poroikov, V. V. (2006). Prediction of biological activity of organic compounds. Rosiiskii himicheskii zhurnal, 2, 66-75.

22. Filimonov, D. A., Poroikov, V. V.; Varnek, A., Tropsha, A. (Eds.) (2008). In Chemoinformatics Approaches to Virtual Screening. Cambridge (UK): RSC Publishing, 182-216.

23. Get more information about biological potential of your compounds. Available at: http://www.pharmaexpert.ru/ passonline

24. Berezovskaya, I. V. (2003). Classification of the chemical parameters on acute toxicity of parenteral routes of administration. Khimiko-Farmatsevticheskii Zhurnal, 37 (3), 32-34.

25. Lagunin, A., Zakharov, A., Filimonov, D., Poroikov, V. (2011). QSAR Modelling of Rat Acute Toxicity on the Basis of PASS Prediction. Molecular Informatics, 30 (2-3), 241-250. doi: $10.1002 / \operatorname{minf} .201000151$

26. GUSAR Online. Available at: http://pharmaexpert.ru/ GUSAR/acutoxpredict.html

\section{Дата надходження рукопису 17.02.2016}

Danylchenko Svitlana, Scientific Secretary of scientific and research department, National University of Pharmacy, 53 Pushkinska str., Kharkiv, Ukraine, 61002

E-mail:povstenko@gmail.com

Drushlyak Oleksander, Candidate of chemical sciences, associate professor, Department of Quality Management, National University of Pharmacy, 53 Pushkinska str., Kharkiv, Ukraine, 61002

E-mail: aldry18@hotmail.com 
Kovalenko Sergiy, Doctor of chemical sciences, professor, Department of Organic Chemistry, V. N. Karazin Kharkiv National University, 4 Svobody Sq., Kharkiv, Ukraine, 61022

E-mail: kovalenko.sergiy.m@gmail.com

Kovalenko Svitlana, Candidate of chemical sciences, associate professor, Department of Quality Management, National University of Pharmacy, 53 Pushkinska str., Kharkiv, Ukraine, 61002

E-mail: claire82@mail.ru

\author{
UDC 615.012:615.28]-092.4 \\ DOI: 10.15587/2313-8416.2016.65209
}

\title{
SYNTHESIS OF THE ROW OF NEW FUNCTIONAL DERIVATIVES OF 7-ARYLALKYL -8-HYDRAZINE THEOPHYLLINES
}

\section{(C) D. Korobko}

Hydrazine functional derivatives are widely used in medical practice as remedies applied for pharmacotherapy of depression, infection diseases, hypertension, diabetes, etc. It is worth mentioning that among obtained 7-R-8hydrazine derivatives of 1,3-dimethylxantine promising substances have been identified. Due to the fact that literature sources display only results of occasional studies of the reactions between 7-R-8-hydrazine theophyllines and mono- or dicarbonyle substances, the use of other keto reagents for xanthine bicycle at ${ }^{\text {th }}$ position functionalization will allow to explore synthetic potential of the last one, and with high probability may lead to obtaining original biologically active substances.

Aim. To study types of reaction between 8- hydrazinyl-1,3-dimethyl-7-aryl alkyl-1H-purine-2,6(3H,7H)-diones and a number of carbonyl containing reagents.

Methods. A nucleophilic addition reaction followed by dehydration or ethanol splitting was used, as well as the complex of the modern analysis methods to confirm the structure and individuality of the synthesized substances. Results. Different directions of 8-hydrazinyl-1,3,-dimethyl-7(fenetyl-, 3-phenylpropyl-, 3-phenylalyl)- 1H-purine$2,6(3 H, 7 H)$-diones chemical transformations in reactions with the appropriate carbonyl containing compounds have been studied experimentally. The structure of synthesized substances was confirmed by chromatography/mass and ${ }^{l} H$ NMR spectroscopy.

Conclusion. The group of 7-arylalkyl-8-(3,5-R, $R_{1}$-pyrazole-1-yl)theophyllines, consisting of two functionally substituted bioactive heterocycles, has been synthesized by reaction between initial substances and selected mono- and dicarbonyl compounds

Keywords: synthesis, 7,8-disubstituted of 1,3-dimethylxantine, hydrazine derivatives, spectral analysis methods

Функціональні похідні гідразину набули широкого застосування в медичній практиці як лікарські засоби для фармакокорекиії депресій, інфекиійних уражень, запальних процесів, гіпертензивних станів, иукрового діабету тощчо. Варто зазначити, щчо серед вже одержаних 7-R-8-гідразинопохідних 1,3диметилксантину також ідентифіковані перспективні у фармакологічному відношенні субстаниії. Оскільки з літератури відомо лише про спорадичні дослідження взаємодії 7-R-8-гідразинотеофілінів 3 моно- та дикарбонільними сполуками, використання інших кетовмісних реагентів з метою функціоналізації 8 положення ксантинового біциклу дозволить не тільки вивчити синтетичний потенціал останнього, але може з високою вирогідністю призвести до одержання нових біологічно активних речовин.

Мета. Дослідити напрямки взаємодії 8-гідразиніл-1,3-диметил-7-арилалкіл-1Н-пурин-2,6(3Н,7Н)-діонів 3 рядом карбонілвмісних реагентів.

Методи. Використано реакцію нуклеофільного приєднання з наступною дегідратацією чи відщепленням етанолу, а також комплекс сучасних спектральних методів аналізу для підтвердження структури й індивідуальності синтезованих речовин.

Результати. Експериментально встановлені окремі напрямки хімічних перетворень 8-гідразиніл-1,3диметил-7-(фенетил-, 3-фенілпропіл-, 3-фенілаліл)-1Н-пурин-2,6(3Н,7Н)-діонів в реакціях з відповідними карбонілвмісними сполуками. Структуру синтезованих речовин підтверджено даними хромато-масma ${ }^{1}$ Н ЯМР-спектрів.

Висновки. Ряд 7-арилалкіл-8-(3,5-R, $R_{1}$-піразол-1-іл)теофілінів, щзо складаються з двох функиіональнозаміщених біоактивних гетероциклів, синтезовані шляхом взаємодії вихідних речовин з окремими представниками моно- та дикарбонільних сполук

Ключові слова: синтез, 7,8-дизаміщені 1,3-диметилксантину, похідні гідразину, спектральні методи аналізу 